\title{
Chirality of matter shows up via spin excitations
}

\author{
S. Bordács ${ }^{1,2}$, I. Kézsmárki ${ }^{1,2 \star}$, D. Szaller ${ }^{1}$, L. Demkón ${ }^{1,2}$, N. Kida ${ }^{2,3}$, H. Murakawa ${ }^{2}$, Y. Onose ${ }^{2,4}$, \\ R. Shimano ${ }^{2,5}$, T. Rõõm ${ }^{6}$, U. Nagel ${ }^{6}$, S. Miyahara ${ }^{2}$, N. Furukawa ${ }^{2,7}$ and Y. Tokura ${ }^{2,4,8}$
}

An object is considered chiral if its mirror image cannot be brought to coincide with itself by any sequence of simple rotations and translations ${ }^{1}$. Chirality on a microscopic scale-in molecules ${ }^{2,3}$, clusters ${ }^{4}$, crystals ${ }^{5}$ and metamaterials ${ }^{6,7}$-can be detected by differences in the optical response of a substance to right- and left-handed circularly polarized light ${ }^{2,3}$. Such 'optical activity' is generally considered to be a consequence of the specific distribution of electronic charge within chiral materials. Here, we demonstrate that a similar response can also arise as a result of spin excitations in a magnetic material. Besides this spin-mediated optical activity (SOA), we observe notable differences in the response of $\mathrm{Ba}_{2} \mathrm{CoGe}_{2} \mathrm{O}_{7}-$ a squarelattice antiferromagnet that undergoes a magnetic-field driven transition to a chiral form-to terahertz radiation travelling parallel or antiparallel to an applied magnetic field. At certain frequencies the strength of this magneto-chiral effect ${ }^{8-10}$ is almost complete, with the difference between parallel and antiparallel absorption of the material approaching $100 \%$. We attribute these phenomena to the magnetoelectric ${ }^{11,12}$ nature of spin excitations as they interact with the electric and magnetic components of light.

Natural circular dichroism (NCD) and gyrotropy are observed respectively as the change in the ellipticity and the rotation of the polarization of light transmitted through chiral media. Because the sign of these quantities depends on the handedness of the material, NCD is a common probe of chirality over a broad spectrum of the electromagnetic radiation, as reviewed in Fig. 1, when applied to the chiroptical study of proteins. Ultraviolet NCD spectroscopy of peptide-bond excitations is a well-established method for the determination of the secondary structure of proteins ${ }^{3,13}$. Recent extensions of NCD spectroscopy to the infrared and X-ray regions have shed light on new signatures of chirality of matter expressed by molecular vibrations $s^{2,5,14}$ and core electron excitations $s^{15}$. Extending this context, the handedness of magnetic matter should also be detected in the NCD spectra of spin excitations, typically at gigahertz to terahertz frequencies, although these have seldom been investigated.

When chirality is accompanied by magnetism, an intriguing optical cross effect, the magneto-chiral dichroism ${ }^{8,9}(\mathrm{MChD})$, emerges besides the conventional magnetically induced circular dichroism (MCD). MChD is a directional dichroism and is measured as the absorption difference for unpolarized (or linearly polarized) light propagating parallel and antiparallel to the magnetization of the media. MChD is generally recognized as a weak effect and has been found for visible light in metallic complexes ${ }^{10}$, molecular magnets ${ }^{16}$, inorganic crystals ${ }^{17}$ and cholesteric liquid crystals ${ }^{18}$. However, a

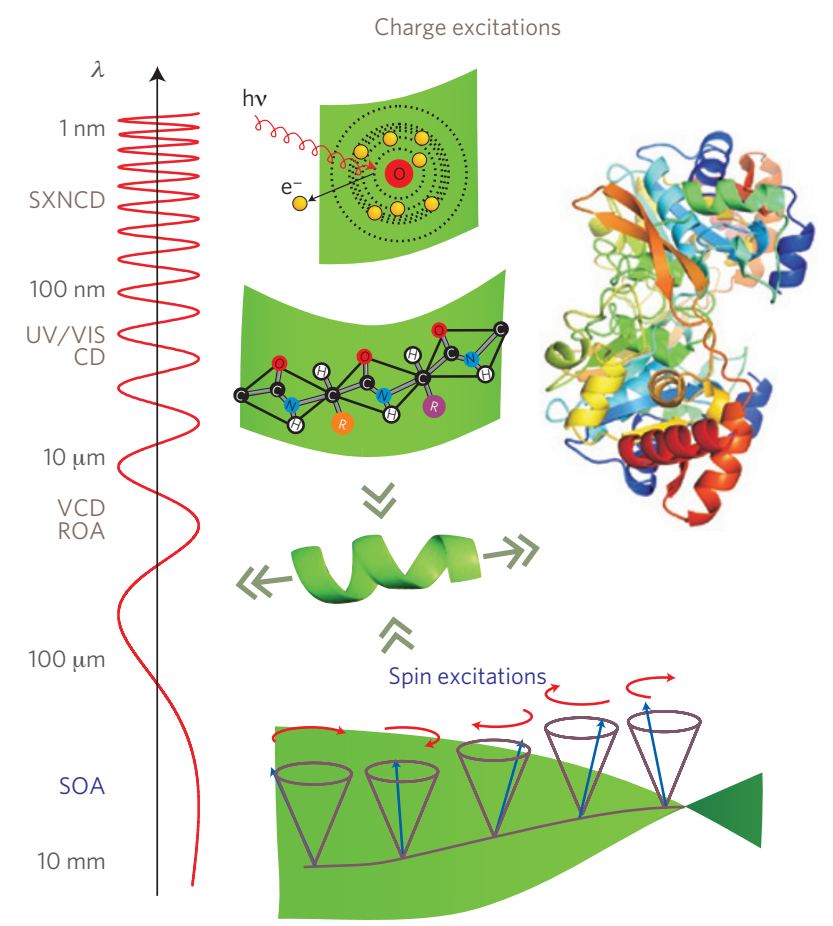

Figure 1 | Chiroptical spectroscopy: an efficient probe of chirality both via charge and spin excitations. Depending on the wavelength, $\lambda$, light interacts with various degrees of freedom and detects the handedness of matter at different levels. The soft X-ray natural circular dichroism (SXNCD) picks up chirality via the core-electron excitations, whereas the circular dichroism in the ultraviolet and visible region (UV/VIS CD) probes it through transitions of valence electrons. Molecular vibrations are also sensitive to the handedness, which is manifested in the vibrational circular dichroism (VCD) or the Raman optical activity (ROA). We predict that besides the charge excitations above, spin-wave excitations in the gigahertz-terahertz region $(\lambda \sim 100 \mu \mathrm{m}-10 \mathrm{~mm})$ of the electromagnetic spectrum can also probe the chirality of magnetic materials and show SOA.

recent study of the chiral phase of $\mathrm{CuB}_{2} \mathrm{O}_{4}$ proved that $\mathrm{MChD}$ can reach the order of unity $(\sim 100 \%)$ in crystal field transitions of $d$ shell electrons ${ }^{19}$. An optical second harmonic generation study of the multiferroic $\mathrm{LiCoPO}_{4}$ also indicated that the electric dipole excitations of the $d$ shell electrons can probe chirality ${ }^{20}$.

Spin-waves (magnons) in conventional magnets are excited by the magnetic component of light and they naturally show the MCD

\footnotetext{
${ }^{1}$ Department of Physics, Budapest University of Technology and Economics and Condensed Matter Research Group of the Hungarian Academy of Sciences, 1111 Budapest, Hungary, ${ }^{2}$ Multiferroics Project, ERATO, Japan Science and Technology Agency (JST), Japan c/o The University of Tokyo, Tokyo 113-8656, Japan, ${ }^{3}$ Department of Advanced Materials Science, The University of Tokyo, 5-1-5 Kashiwanoha, Chiba 277-8561, Japan, ${ }^{4}$ Department of Applied Physics, The University of Tokyo, Tokyo 113-8656, Japan, ${ }^{5}$ Department of Physics, The University of Tokyo, Tokyo 113-0033, Japan, ${ }^{6}$ National Institute of Chemical Physics and Biophysics, Tallinn 12618, Estonia, ${ }^{7}$ Department of Physics and Mathematics, Aoyama Gakuin University, 5-10-1 Fuchinobe, Chuo-ku, Sagamihara, Kanagawa 252-5258, Japan, ${ }^{8}$ Cross-correlated Materials Group (CMRG) and Correlated Electron Research Group (CERG), RIKEN Advanced Science Institute, Wako 351-0198, Japan. *e-mail: kezsmark@dept.phy.bme.hu.
} 


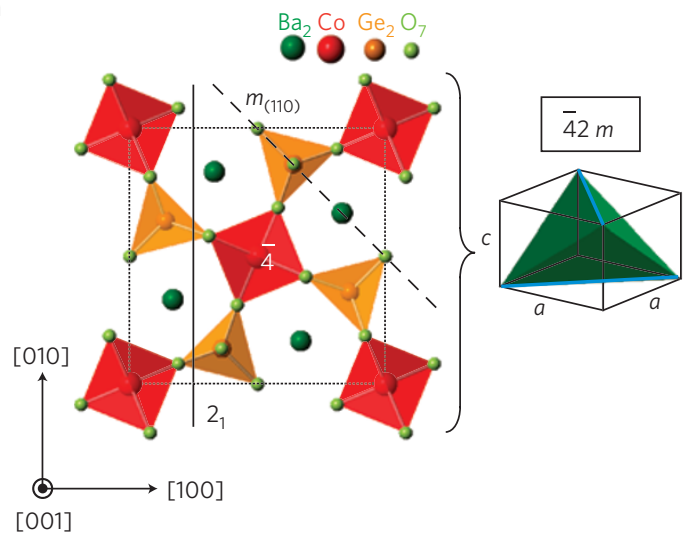

b

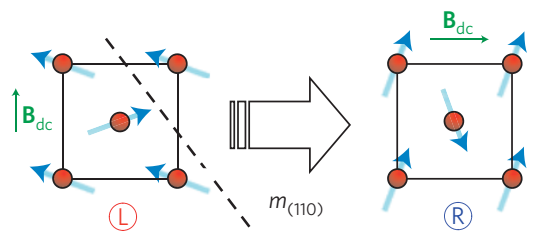

C

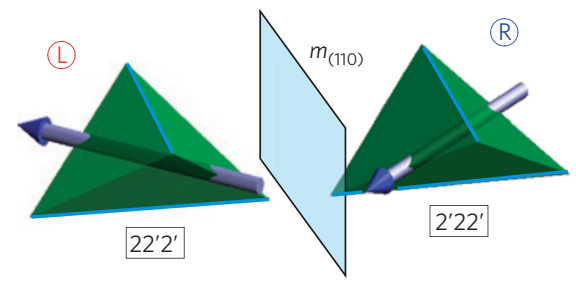

Figure 2 | Main aspects of magnetically induced chirality. a, The crystal structure of $\mathrm{Ba}_{2} \mathrm{CoGe}_{2} \mathrm{O}_{7}$ belongs to the tetragonal space group $P \overline{4} 2{ }_{1} m$. The corresponding $\overline{4} 2 \mathrm{~m}$ point group can be represented by a single tetrahedron compressed in the [001] direction. Its two longer edges are indicated with blue lines. b. When the external magnetic field points along the [100] or [010] axis, the antiferromagnetic spin pattern with field-induced canting (blue arrows) breaks all mirror-plane symmetries of the lattice, hence, it makes the crystal chiral with the point symmetry of $22^{\prime} 2^{\prime}$ or $2^{\prime} 22^{\prime}$, respectively. Switching between the left-handed $(L)$ and right-handed $(R)$ enantiomers can be carried out by rotation of the magnetic field from the [100] to the [010] direction, being equivalent to the $m_{(110)}$ mirror reflection (dashed line). c, The magnetically induced chirality of the material and the corresponding point symmetries can be schematized by the compressed tetrahedron together with a magnetic moment connecting midpoints of two opposite shorter edges (representing the lattice symmetry and the spin system, respectively). In the mirror image obtained by $m_{(110)}$ reflection, the tetrahedron remains unchanged, while the magnetic moment (axial vector) is rotated by $\pi / 2$. Image and mirror image are enantiomeric pairs as they cannot be brought into coincidence with each other by pure rotations and translations even when combined with time-reversal operation.
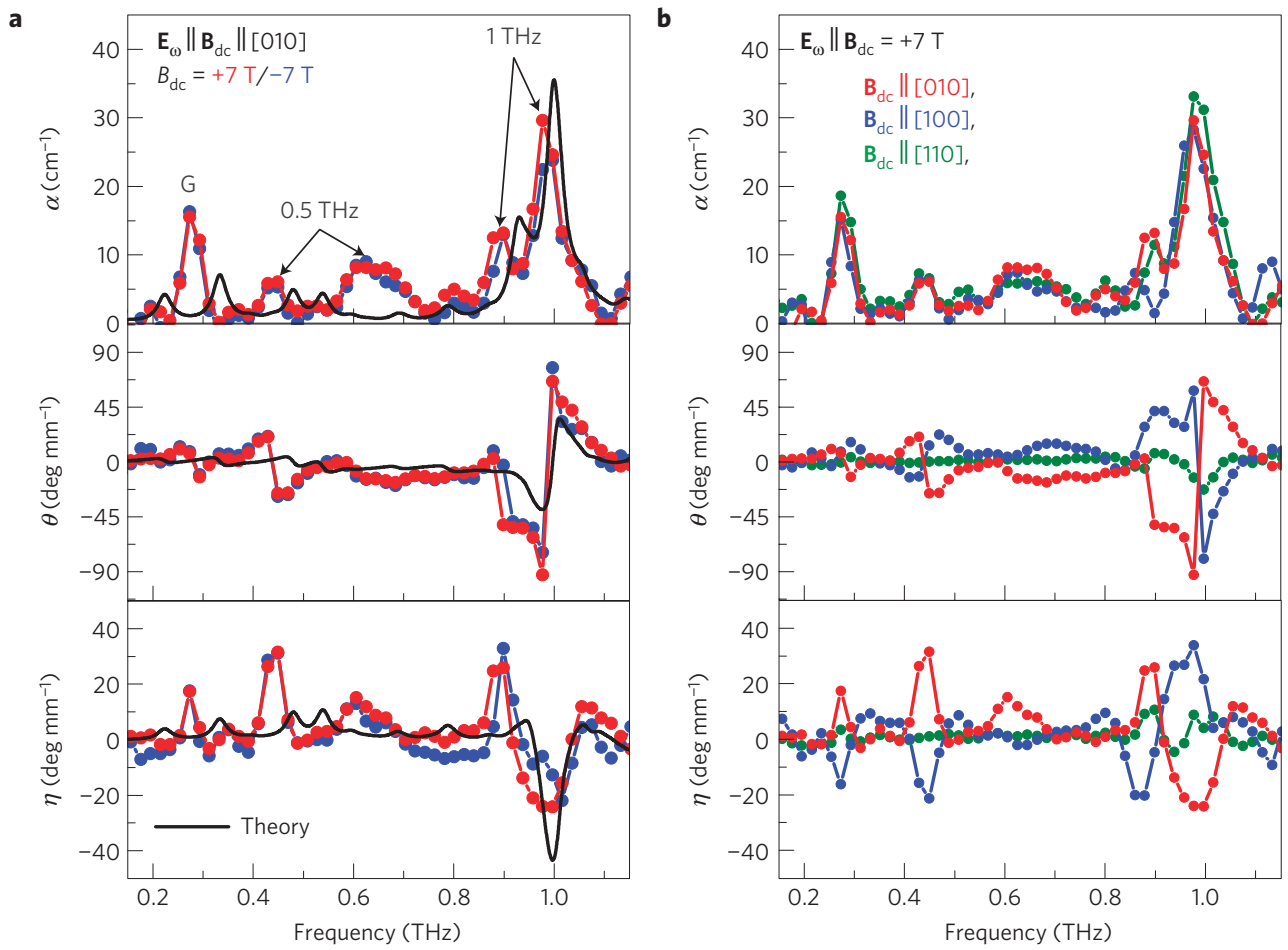

Figure 3 | Absorption $(\alpha)$, polarization rotation $(\theta)$ and ellipticity $(\eta)$ spectra of the spin-wave modes for light propagation along the [001] axis as measured by time-domain terahertz spectroscopy and calculated theoretically. a, The modes observed at $0.5 \mathrm{THz}$ and $1 \mathrm{THz}$ in zero field show clear splittings in high magnetic fields parallel to the [010] axis, as indicated by arrows. The 'Goldstone mode' (G) is centred around $0.27 \mathrm{THz}$. The large polarization rotation and ellipticity are even functions of the magnetic field. $\mathbf{b}$, When the external magnetic field direction is rotated from the [100] to the [010] axis, both $\theta$ and $\eta$ change sign, whereas they are zero within the experimental accuracy when the magnetic field points along the [110] direction and the $m_{(110)}$ mirror-plane symmetry is restored. Thus, these polarization phenomena can be identified as SOA in a chiral media.

effect, whereas NCD and MChD effects are forbidden. A prerequisite to observe such phenomena in the long-wavelength region is the existence of dynamical coupling between spins and microscopic electric dipoles in the material' ${ }^{2}$. In multiferroic materials, where magnetization $(M)$ and ferroelectric polarization $(P)$ are inter- locked, such cross-coupling is possible and was indeed observed as electrically excited magnetic resonance in several compounds, including $\mathrm{RMnO}_{3}$ (refs 21,22), $\mathrm{RMn}_{2} \mathrm{O}_{5}$ (ref. 23), $\mathrm{BiFeO}_{3}$ (ref. 24), and most recently in $\mathrm{Ba}_{2} \mathrm{CoGe}_{2} \mathrm{O}_{7}$ (ref. 25). However, the question of chirality has not been addressed so far in these systems. We pre- 

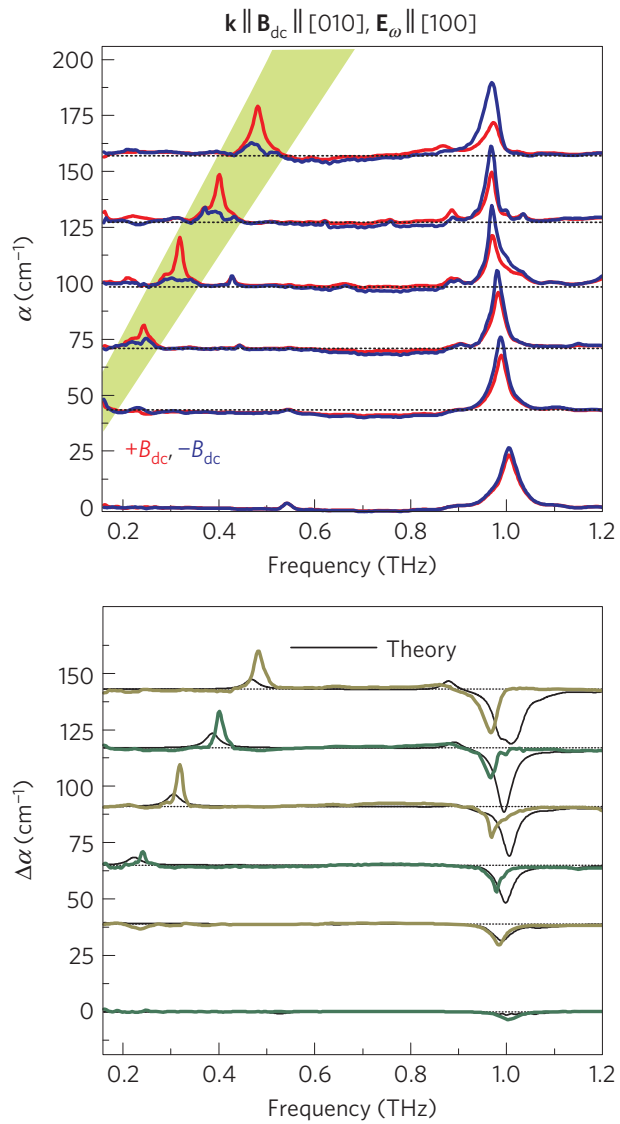
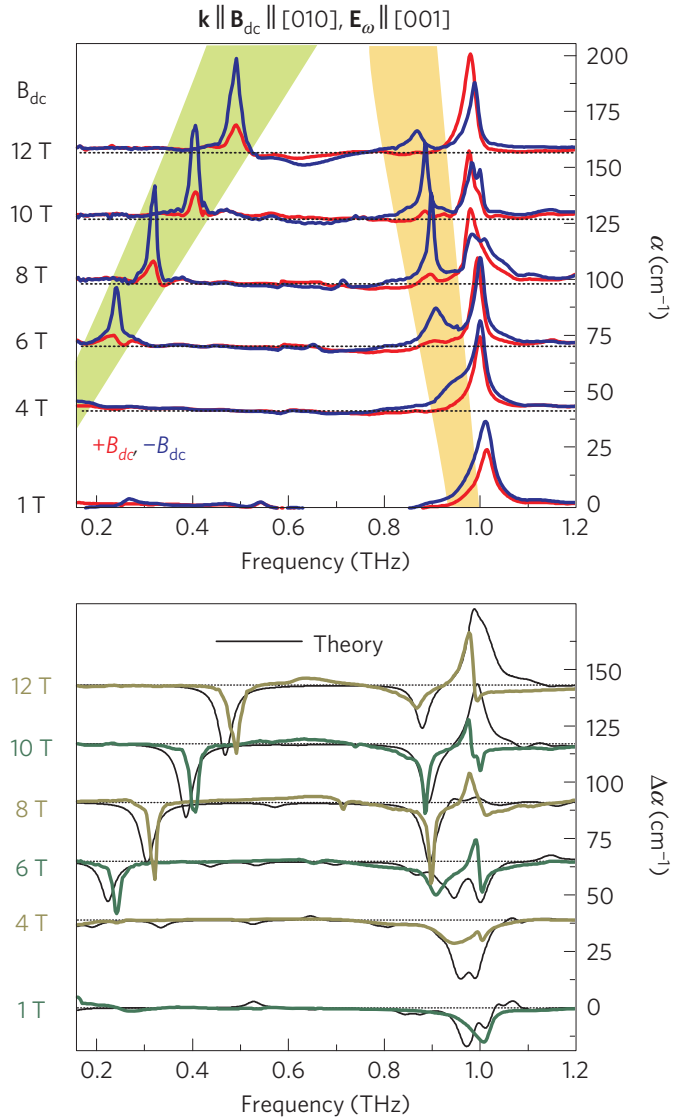

Figure 4 | Magneto-chiral effect in the field-induced chiral state. Absorption is studied when light propagates along the $[010]$ axis parallel $\left(+B_{\mathrm{dc}}\right)$ and antiparallel $\left(-B_{\mathrm{dc}}\right)$ to the external magnetic field. $\mathbf{a}, \mathbf{b}$, Absorption spectra for light polarizations $\mathbf{E}_{\omega} \|[100]$ (a) and $\mathbf{E}_{\omega} \|[001](\mathbf{b})$, measured as a function of the magnetic field, are shifted in proportion to the corresponding $B_{\mathrm{dc}}$ values. Red and blue curves indicate the case of $+B_{\mathrm{dc}}$ and $-B_{\mathrm{dc}}$, respectively. Between the spin resonances the material is transparent, as $\alpha \approx 0$. The magneto-chiral dichroism, corresponding to the difference of the absorption coefficients for counter-propagating beams, is huge both for the 'Goldstone mode' (highlighted green) and the low-frequency branch of the resonance at $\sim 1 \mathrm{THz}$ (highlighted orange). $\Delta \alpha / \alpha_{\max } \gtrsim 70 \%$ in the highlighted regions. c,d, The sign and magnitude of the experimentally observed MChD effect (coloured curves) are reproduced by our theoretical model (black curves) for two polarizations $\mathbf{E}_{\omega} \|[100]$ and $\mathbf{E}_{\omega} \|[100]$ as in $\mathbf{c}$ and $\mathbf{d}$, respectively.

dict that a magnetically induced chiral state with switchable handedness can emerge in magnets with non-centrosymmetric but nonchiral crystal structure, as we demonstrate for $\mathrm{Ba}_{2} \mathrm{CoGe}_{2} \mathrm{O}_{7}$. Crossresonances between electric and magnetic dipole active transitions existing at spin excitations in $\mathrm{Ba}_{2} \mathrm{CoGe}_{2} \mathrm{O}_{7}$ provide us with an excellent opportunity to observe gigantic NCD and MChD effects.

$\mathrm{Ba}_{2} \mathrm{CoGe}_{2} \mathrm{O}_{7}$ has a tetragonal crystal structure where $\mathrm{Co}^{2+}$ cations with $S=3 / 2$ spin form square-lattice layers stacked along the tetragonal [001] axis (see Fig. 2a). Néel-type antiferromagnetic order develops below $T_{\mathrm{N}}=6.7 \mathrm{~K}$, with spins lying in the plane of the layers (tetragonal plane) ${ }^{26}$. Moderate magnetic fields, $B_{\mathrm{dc}} \gtrsim 1 \mathrm{~T}$, applied in this plane effectively induce a ferromagnetic moment by canting the spins, while the antiferromagnetic component of the magnetization aligns perpendicular to the field, as shown in Fig. 2b. When the ferromagnetic moment points along the [110] axis, a ferroelectric polarization appears parallel to the [001] axis $^{27}$. Rotation of the magnetic field within the tetragonal plane rotates $M$, accompanied by the sinusoidal modulation of $P$ (ref. 27). Whereas ferroelectricity vanishes in magnetic fields applied along the [100] or [010] axis, the corresponding spin patterns break all mirror-plane symmetries of the lattice, as visualized in Fig. 2b,c, and the material becomes chiral ${ }^{19,28}$. We emphasize that although neither the spin nor the lattice structure alone is chiral, the whole spin-lattice system forms a chiral object: the $\pi / 2$ rotation of the magnetic field from [100] to [010] direction switches between the left- and right-handed forms of the magnetic crystal, corresponding to the chiral space groups $P 2_{1} 2_{1}^{\prime} 2^{\prime}$ and $P 2_{1}^{\prime} 2_{1} 2^{\prime}$, respectively ${ }^{28}$ (see Fig. $2 b, c)$. Such soft switching of handedness is hardly conceivable in molecules and crystals with purely structural chirality.

The nature of the spin excitations in the magnetically induced chiral state of $\mathrm{Ba}_{2} \mathrm{CoGe}_{2} \mathrm{O}_{7}$ was studied by polarization-sensitive optical spectroscopy over the gigahertz-terahertz regime (see Methods). We found two spin resonances located at frequencies $f \approx 0.5 \mathrm{THz}$ and $f \approx 1 \mathrm{THz}$ in zero field. With increasing magnetic field a third mode appears from low frequencies, which corresponds to the gapless Goldstone mode in the zero-field limit ${ }^{26}$. These modes exhibit strong polarization rotation and ellipticity for each propagation direction and polarization configuration whenever the static magnetic field, $\mathbf{B}_{\mathrm{dc}}$, points along [100] or [010] direction. (The full polarization study is presented in the Supplementary Information.) When the light propagates along the [001] axis perpendicular to the static magnetic field and $\mathbf{E}_{\omega} \| \mathbf{B}_{\mathrm{dc}}$, as in a typical case shown in Fig. 3a, all three resonances are excited. In $B_{\mathrm{dc}}=7 \mathrm{~T}$, the 'Goldstone mode' is located at $f \approx 0.27 \mathrm{THz}$ and the other two modes are split into several bands. The band around $1 \mathrm{THz}$ induces a strong polarization change characterized by $\theta \approx 90^{\circ} \mathrm{mm}^{-1}$, $\eta \approx 35^{\circ} \mathrm{mm}^{-1}$ close to the resonance frequency, whereas the effect is a factor of three smaller for the lower-frequency magnon modes.

These polarization phenomena are even functions of the magnetic field, thereby excluding any contributions from conventional MCD. Furthermore, the sign of the polarization rotation and ellipticity spectra are reversed by the simultaneous rotation of $\mathbf{B}_{\mathrm{dc}}$ 
and $\mathbf{E}_{\omega}$ from [100] to [010], as shown in Fig. 3b. In the case of magnetically induced linear birefringence/dichroism, the optical axes would be linked to the direction of $\mathbf{B}_{\mathrm{dc}}$ as the (001) plane is originally isotropic in the paramagnetic state. As $\mathbf{E}_{\omega}$ is kept parallel with $\mathbf{B}_{\mathrm{dc}}$, the sign change observed in $\theta$ and $\eta$ is not compatible with linear birefringence and dichroism, although the magnitude of the rotation and ellipticity spectra can be influenced by these linear polarization effects (see Supplementary Information). Instead, this sign reversal is specific to optical activity and provides a direct proof of the true chirality and the field-induced switching of the handedness of matter. In fields applied along the intermediate [110] direction, by contrast, no NCD and gyrotropy appear, indicating the lack of chirality as the $m_{(110)}$ mirror-plane symmetry is restored.

The existence of $\mathrm{MChD}$ was investigated in the chiral state by keeping $\mathbf{B}_{\mathrm{dc}}$ along the [010] or [100] axis, while choosing the light propagation to be parallel or antiparallel to $\mathbf{B}_{\mathrm{dc}}$ (see Fig. 4). In this configuration, called the Faraday geometry, the magnetic resonance at $\sim 0.5 \mathrm{THz}$ is silent. The mode at $1 \mathrm{THz}$ is split in larger fields and the 'Goldstone mode' enters from lower frequencies above $4 \mathrm{~T}$. The magnetically induced handedness is also manifested in strong NCD and gyrotropy (see Supplementary Information).

The difference in absorption, $\Delta \alpha=\alpha^{+}-\alpha^{-}$, for light propagating parallel and antiparallel to the external field is large for all optically active spin resonances (Fig. 4c,d). Note that the 'Goldstone mode' exhibits MChD as large as $\Delta \alpha / \alpha_{\max } \gtrsim 70 \%$ for both polarizations in high fields, where $\alpha_{\max }=\max \left\{\alpha^{+}, \alpha^{-}\right\}$. For the light polarization $\mathbf{E}_{\omega} \|[001]$ shown in Fig. 4, the absorption nearly vanishes at the low-frequency branch of the $1 \mathrm{THz}$ resonance for the beam propagating antiparallel to the direction of $B_{\mathrm{dc}}$. It means that the material becomes transparent at these frequencies when viewed from the direction of the magnetization, whereas the absorption remains fairly large for light travelling in the opposite direction, that is, $\Delta \alpha / \alpha_{\max }$ is close to $100 \%$. As is clear from Fig. $3 \mathrm{c}, \mathrm{d}$, the effect is still appreciable in low magnetic fields.

$\mathrm{NCD}$ and $\mathrm{MChD}$, emerging in $\mathrm{Ba}_{2} \mathrm{CoGe}_{2} \mathrm{O}_{7}$ with large magnitude in the gigahertz-terahertz range, are observed for the first time for spin excitations. In the conventional view spins respond to the magnetic field of light, which induces their precession described by the dynamical magnetic permeability $\hat{\mu}(\omega)$. In this multiferroic material, where spins are coupled to microscopic electric dipoles, a new channel opens to excite the spins via the electric field of light ${ }^{29}$, the so-called electromagnon process. Consequently, the dynamics of the spins also induces oscillating electric polarization in the material, expressed by the dielectric permittivity $\hat{\varepsilon}(\omega)$.

Because these spin resonances can simultaneously be excited by the magnetic and electric components of light with similar strength, the dynamical magnetoelectric tensor $\hat{\chi}^{\mathrm{me}}(\omega)$ also affects the optical response. It describes the oscillations of the magnetization in the material induced by the electric field of light, $\Delta \mathbf{M}_{\omega}=\sqrt{\left(\varepsilon_{0} / \mu_{0}\right)} \hat{\chi}^{\mathrm{me}}(\omega) \mathbf{E}_{\omega}$, in analogy with the d.c. magnetoelectric effect $\left(\varepsilon_{0}\right.$ and $\mu_{0}$ are the dielectric permittivity and magnetic permeability of vacuum). In $\mathrm{Ba}_{2} \mathrm{CoGe}_{2} \mathrm{O}_{7}$, the symmetry of the magnetically ordered phase and the corresponding form of $\hat{\chi}^{\text {me }}$ can be controlled by the magnetic field. Specifically, fields $B_{\mathrm{dc}} \gtrsim$ $1 \mathrm{~T}$ applied parallel to the [100] or [010] crystallographic axis yield

$$
\hat{\chi}_{[100]}^{\mathrm{me}}=-\hat{\chi}_{[010]}^{\mathrm{me}}=\left(\begin{array}{ccc}
\chi_{x x}^{\mathrm{me}} & 0 & \chi_{x z}^{\mathrm{me}} \\
0 & \chi_{y y}^{\mathrm{me}} & 0 \\
\chi_{z x}^{\mathrm{me}} & 0 & \chi_{z z}^{\mathrm{me}}
\end{array}\right)
$$

where $\mathbf{B}_{\mathrm{dc}}$ and the [001] tetragonal axis are set parallel to the $y$ - and the $z$-axis, respectively. The sign change of $\hat{\chi}^{\text {me }}$ on $\pi / 2$ rotation of the crystal around the $z$-axis is related to the switching between the two enantiomers. The diagonal components (invariant under time-reversal) are responsible for NCD, whereas the off-diagonal elements (changing sign on time-reversal) generate $\mathrm{MChD}$. The existence of magnetically induced chirality and NCD in $\mathrm{Ba}_{2} \mathrm{CoGe}_{2} \mathrm{O}_{7}$ can alternatively be predicted by the symmetry analysis of the magneto-gyration tensor $F_{\alpha \beta \gamma \delta}$ in its paramagnetic space group $P \overline{4} 2_{1} m$. This response function is related to the magnetoelectric tensor according to $F_{\alpha \beta \gamma \delta}=\left(\partial^{2} g_{\alpha \beta}\right) /\left.\left(\partial B_{\gamma} \partial B_{\delta}\right)\right|_{B=0}=$ $1 / c\left(\partial^{2}\left(\chi_{\beta \alpha}^{\mathrm{me}}-\chi_{\gamma \gamma}^{\mathrm{me}} \cdot \delta_{\alpha \beta}\right)\right) /\left.\left(\partial B_{\gamma} \partial B_{\delta}\right)\right|_{B=0}$, where $g_{\alpha \beta}$ is the gyration tensor, $B$ is the magnetic field and $c$ is the speed of light ${ }^{2}$. Here the Greek symbols stand for cartesian indices $x, y$ and $z$ while $\delta$ denotes Kronecker delta. In accordance with the observed sign change of NCD on rotation of $\mathbf{B}_{\mathrm{dc}}$ from the [100] to the [010] direction, Neumann's principle gives that $F_{z z x x}$ and $F_{z z y y}$ components are finite $\left(F_{z z x x}=-F_{z z y y}\right)$, while the lack of NCD for $B_{\mathrm{dc}} \|[110]$ corresponds to $F_{z z x y}=0$.

We have successfully described the magnetically induced chirality in the ground state of $\mathrm{Ba}_{2} \mathrm{CoGe}_{2} \mathrm{O}_{7}$ and its spin excitation spectrum based on a microscopic spin model. Next, we solved the Maxwell equations using $\hat{\mu}(\omega), \hat{\varepsilon}(\omega)$ and $\hat{\chi}^{\mathrm{me}}(\omega)$ obtained from this theory. (For details see the Supplementary Information.) The calculated absorption and gyrotropy/NCD spectra-as approximated by $\alpha^{ \pm}=(2 \omega / c) \mathfrak{s}\left\{\sqrt{\varepsilon_{\gamma \gamma} \mu_{\delta \delta}} \pm \chi_{\gamma \delta}^{\text {me }}\right\}$ and $\theta+i \eta=(i \omega / 2 c)\left\{\chi_{\gamma \gamma}^{\text {me }}+\chi_{\delta \delta}^{\text {me }}\right\}$, respectively-reproduce the experimental results as shown in Fig. 3. Furthermore, the measured MChD spectra shown in Fig. 4c,d show an overall agreement with the theoretical curves $\Delta \alpha=\alpha^{+}-\alpha^{-}=(4 \omega) /(c) \operatorname{Im}\left\{\chi_{\gamma \delta}^{\text {me }}\right\}$.

The observed strong NCD and gyrotropy-what we call SOA - together with the giant $\mathrm{MChD}$ are the direct manifestations of the dynamical magnetoelectric effect. Because simple spin orders, such as ferromagnetic or antiferromagnetic, can effectively generate magnetoelectric coupling in crystals without inversion symmetry, we expect that a broad variety of magnetic materials offer these novel optical functionalities for the polarization and directional control of light.

\section{Methods}

Samples. Single crystals of $\mathrm{Ba}_{2} \mathrm{CoGe}_{2} \mathrm{O}_{7}$ were grown by a floating zone method. Crystals were characterized by ferroelectric polarization and magnetization measurements. The precisely oriented samples used in the terahertz study had slab-like shapes with a typical thickness of $1 \mathrm{~mm}$ and cross-section of $10 \mathrm{~mm}^{2}$.

Circular dichroism spectroscopy. We adopted terahertz time-domain spectroscopy $y^{30,31}$ to obtain the complex transmission spectrum in the frequency range $0.2-2 \mathrm{THz}$ with a resolution of $0.05 \mathrm{THz}$. The femtosecond laser pulses delivered from a mode-locked Ti:sapphire laser (centre wavelength of $800 \mathrm{~nm}$, pulse width of $100 \mathrm{fs}$, repetition rate of $80 \mathrm{MHz}$ ) were irradiated to a biased photo-switching device made of low-temperature-grown GaAs (LT-GaAs) coupled with a dipole antenna, or to the surface of a p-type InAs crystal. The terahertz pulses radiated by such emitters were collimated by a pair of off-axis paraboloidal mirrors and were focused on the sample. After passing through the sample the beam was collimated again and focused on the detector by a second pair of off-axis paraboloidal mirrors. For the detection, another LT-GaAs photo-switching device coupled with a dipole antenna or an electro-optic sampling method with a (110) ZnTe crystal was used. The light polarization was carefully set parallel to each crystallographic axis using a wire-grid polarizer. The polarization state of the beam transmitted through the sample was analysed by another wire-grid polarizer. To obtain the absolute transmission of the material, the sample was replaced by a hole with the same diameter, through which the direct beam was measured. Static magnetic fields up to $\pm 7 \mathrm{~T}$ were applied by a superconducting split-coil magnet.

From the time-domain transmission experiments, performed in several polarization configurations, we determined each element of the complex $2 \times 2 \hat{t}_{i j}$ transmission matrix. Besides the absorption coefficient, $\alpha \approx(-1 / d) \ln \left(\left|\tilde{t}_{x x}\right|^{2}+\left|\tilde{t}_{y x}\right|^{2}\right)$, changes in the polarization state of linearly polarized light due to NCD and gyrotropy were determined according to

$$
\frac{\tan \theta+i \tan \eta}{1-i \tan \theta \tan \eta}=\frac{\tilde{t}_{y x}}{\tilde{t}_{x x}}
$$

where $d$ is the sample thickness, $\tilde{t}_{x x}$ and $\tilde{t}_{y x}$ are the complex transmission coefficients for the sample placed between parallel and crossed polarizers, while $\theta$ and $\eta$ are the polarization rotation and ellipticity, respectively (for more details see Supplementary Information). 
Magnetochiral dichroism experiment. For the study of the MChD effect, we used Fourier transform absorption spectroscopy. The measurement system, which is based on a Martin-Puplett interferometer, a mercury arc lamp as a light source, and a Si bolometer cooled down to $300 \mathrm{mK}$ as a light intensity detector, covered the spectral range $0.13-6 \mathrm{THz}$ with a maximum resolution of $0.004 \mathrm{THz}$. A single wire-grid polarizer was inserted to set the polarization state of the beam before the sample. However, the detection side, that is, the light path after the sample including the detector, was insensitive to the polarization of light. Static magnetic fields up to $\pm 12 \mathrm{~T}$ were applied by a superconducting solenoid magnet.

Received 3 August 2011; accepted 11 July 2012; published online 26 August 2012

\section{References}

1. Kelvin, L. Baltimore Lectures on the Molecular Dynamics and the Wave Theory of Light (C.J. Clay \& Sons, 1904).

2. Barron, L. D. Molecular Light Scattering and Optical Activity (Cambridge Univ. Press, 2004)

3. Berova, N., Nakanishi, K. \& Woody, R. W. Circular Dichroism: Principles and Applications 2nd edn (Wiley-VCH, 2000).

4. Micali, N. et al. Selection of supramolecular chirality by application of rotational and magnetic forces. Nature Chem. 4, 201-207 (2012).

5. Hsu, E. C. \& Holzwarth, G. Vibrational circular dichroism observed in crystalline $\alpha$-NiSO $\cdot 6 \mathrm{HO}$ and $\alpha$-ZnSeO $\cdot 6 \mathrm{HO}$ between 1900 and $5000 \mathrm{~cm}^{-1}$. J. Chem. Phys. 59, 4678-4685 (1973).

6. Kuwata-Gonokami, M. et al. Giant optical activity in quasi-two-dimensional planar nanostructures. Phys. Rev. Lett. 95, 227401 (2005).

7. Hendry, E. et al. Ultrasensitive detection and characterization of biomolecules using superchiral fields. Nature Nanotech. 5, 783-787 (2010).

8. Baranova, N. B. \& Zeldovich, B. Ya. Theory of a new linear magnetorefractive effect in liquids. Mol. Phys. 38, 1085-1098 (1979).

9. Barron, L. D. \& Vrbancich, J. Magneto-chiral birefringence and dichroism. Mol. Phys. 51, 715-730 (1984).

10. Rikken, G. L. J. A. \& Raupach, E. Observation of magneto-chiral dichroism. Nature 390, 493-494 (1997).

11. Fiebig, M. Revival of the magnetoelectric effect. J. Phys. D 38, R123-R152 (2005)

12. Spaldin, N. A. \& Fiebig, M. The renaissance of magnetoelectric multiferroics. Science 309, 391-392 (2005)

13. Greenfield, N. J. Using circular dichroism spectra to estimate protein secondary structure. Nature Protocols 1, 2876-2890 (2007).

14. Stephens, P. J., Devlin, F. J. \& Pan, J-J. The determination of the absolute configurations of chiral molecules using vibrational circular dichroism (VCD) spectroscopy. Chirality 20, 643-663 (2008).

15. Alagna, L. et al. X-ray natural circular dichroism. Phys. Rev. Lett. 80, 4799-4802 (1998)

16. Train, C. et al. Strong magneto-chiral dichroism in enantiopure chiral ferromagnets. Nature Mater. 7, 729-734 (2008)

17. Rikken, G. L. J. A. \& Raupach, E. Pure and cascaded magnetochiral anisotropy in optical absorption. Phys. Rev. E 58, 5081-5084 (1998).

18. Koerdt, C., Duchs, G. \& Rikken, G. L. J. A. Magnetochiral anisotropy in Bragg scattering. Phys. Rev. Lett. 91, 073902 (2003).

19. Saito, M., Ishikawa, K., Taniguchi, K. \& Arima, T. Magnetic control of crystal chirality and the existence of a large magneto-optical dichroism effect in $\mathrm{CuB}_{2} \mathrm{O}_{4}$. Phys. Rev. Lett. 101, 117402 (2008).
20. Van Aken, B. B., Rivera, J-P., Schmid, H. \& Fiebig, M. Observation of ferrotoroidic domains. Nature 449, 702-705 (2007).

21. Pimenov, A. et al. Possible evidence for electromagnons in multiferroic manganites. Nature Phys. 2, 97-100 (2006).

22. Kida, N. et al. Terahertz time-domain spectroscopy of electromagnons in multiferroic perovskite manganites. J. Opt. Soc. Am. B 26, A35-A51 (2009).

23. Sushkov, A. B., Aguilar, R. V., Park, S., Cheong, S-W. \& Drew, H. D. Electromagnons in multiferroic $\mathrm{YMn}_{2} \mathrm{O}_{5}$ and $\mathrm{TbMn}_{2} \mathrm{O}_{5}$. Phys. Rev. Lett. 98, 027202 (2007).

24. Rovillain, P. et al. Electric-field control of spin waves at room temperature in multiferroic $\mathrm{BiFeO}_{3}$. Nature Mater. 9, 975-979 (2010).

25. Kézsmárki, I. et al. Enhanced directional dichroism of terahertz light in resonance with magnetic excitations of the multiferroic $\mathrm{Ba}_{2} \mathrm{CoGe}_{2} \mathrm{O}_{7}$ oxide compound. Phys. Rev. Lett. 106, 057403 (2011).

26. Zheludev, A. et al. Spin waves and the origin of commensurate magnetism in $\mathrm{Ba}_{2} \mathrm{CoGe}_{2} \mathrm{O}_{7}$. Phys. Rev. B 68, 024428 (2003).

27. Murakawa, H., Onose, Y., Miyahara, S., Furukawa, N. \& Tokura, Y. Ferroelectricity induced by spin-dependent metal-ligand hybridization in $\mathrm{Ba}_{2} \mathrm{CoGe}_{2} \mathrm{O}_{7}$. Phys. Rev. Lett. 103, 137202 (2010).

28. Perez-Mato, J. M. \& Riberio, J. L. On the symmetry and the signature of atomic mechanisms in multiferroics: the example of $\mathrm{Ba}_{2} \mathrm{CoGe}_{2} \mathrm{O}_{7}$. Acta Crystallogr. A 67, 264-268 (2011).

29. Miyahara, S. \& Furukawa, N. Theory of magnetoelectric resonance in two-dimensional $S=3 / 2$ antiferromagnet $\mathrm{Ba}_{2} \mathrm{CoGe}_{2} \mathrm{O}_{7}$ via spin-dependent metal-ligand hybridization mechanism. J. Phys. Soc. Jpn 80, 073708 (2011).

30. Tonouchi, M. Cutting-edge terahertz technology. Nature Photon. 1, 97-105 (2007).

31. Nuss, M. C. \& Orenstein, J. in Millimeter and Submillimeter Wave Spectroscopy of Solids (ed. Grüner, G.) (Springer, 1998).

\section{Acknowledgements}

We thank T. Arima and K. Penc for discussions. This work was supported by KAKENHI, MEXT of Japan, by Funding Program for World-Leading Innovation R\&D on Science and Technology (FIRST program) on 'Quantum Science on Strong Correlation', by Hungarian Research Funds OTKA PD75615, Bolyai 00256/08/11, TA'MOP-4.2.2.B-10/1-2010-0009 and by the Estonian Ministry of Education and Research under Grant SF0690029s09, and Estonian Science Foundation under Grants ETF8170 and ETF8703.

\section{Author contributions}

S.B., I.K., T.R., U.N., D.S. and L.D. performed the measurements and analysed the data; H.M. and Y.O. contributed to the sample preparation; N.K., R.S., T.R. and U.N. developed the experimental set-up; S.M. and N.F. developed the theory; S.B. and I.K. wrote the manuscript; and Y.T. and I.K. planned the project.

\section{Additional information}

Supplementary information is available in the online version of the paper. Reprints and permissions information is available online at www.nature.com/reprints. Correspondence and requests for materials should be addressed to I.K.

\section{Competing financial interests}

The authors declare no competing financial interests. 